\title{
Psychiatric morbidity and dietary habits during COVID-19 pandemic: a cross- sectional study among Egyptian Youth (14-24 years)
}

Roa Gamal Alamrawy ${ }^{1 *}$ (D) Noha Fadl ${ }^{2}$ (D) and Asmaa Khaled ${ }^{3}$ (D)

\begin{abstract}
Background: The coronavirus disease 2019 (COVID-19) is influencing all segments of society, including youth. Although the physical complaints in the time of COVID-19 are broadly-studied, a paucity of research targeted psychological ones on the precious youth population. This study aimed to describe the real-time state of Egyptian youth's psychiatric morbidity, dietary changes, and coping methods during this pandemic and explore probable factors influencing them. A descriptive cross-sectional study was conducted using an online survey among 447 Egyptian participants aged 14-24 years. Sociodemographic data, dietary habits, and coping methods during COVID19 were collected. The Arabic versions of the Patient Health Questionnaire (PHQ-9), the Generalized Anxiety Disorder Scale (GAD-7), and the Insomnia Severity Index (ISI) were used to assess depression, anxiety and insomnia, respectively.

Results: Overall, $80.5 \%, 74.0 \%$, and $73.8 \%$ of the participants had different grades of depression, anxiety, and insomnia symptoms. 37.4\% gained weight. Emotional and night eating emerged as new habits during the pandemic among $17.9 \%$ and $29.3 \%$ of the participants, respectively. Each of depression, anxiety, and insomnia was significantly associated with each other, female gender and having a COVID-19 infected relative. Adolescents had significantly higher scores of depression and anxiety. Those with a history of physical illness had significantly higher scores of anxiety and insomnia. Bodyweight and dietary changes were significantly associated with depression, anxiety, and insomnia. Participants reported various positive and negative coping methods.

(Continued on next page)
\end{abstract}

\footnotetext{
* Correspondence: Roa.Alamrawy@gmail.com

'Mamoura Psychiatric Hospital, General Secretariat of Mental Health and Addiction Treatment, El Nabawy El Mohandes Street, Mamoura, Alexandria 21912, Egypt

Full list of author information is available at the end of the article
}

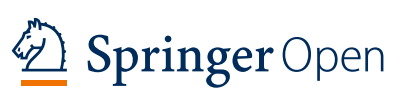

(c) The Author(s). 2021 Open Access This article is licensed under a Creative Commons Attribution 4.0 International License, which permits use, sharing, adaptation, distribution and reproduction in any medium or format, as long as you give appropriate credit to the original author(s) and the source, provide a link to the Creative Commons licence, and indicate if changes were made. The images or other third party material in this article are included in the article's Creative Commons licence, unless indicated otherwise in a credit line to the material. If material is not included in the article's Creative Commons licence and your intended use is not permitted by statutory regulation or exceeds the permitted use, you will need to obtain permission directly from the copyright holder. To view a copy of this licence, visit http://creativecommons.org/licenses/by/4.0/. 
(Continued from previous page)

Conclusion: Psychiatric morbidity and dietary changes are evident in young people during COVID-19 pandemic. Psychological well-being and dietary habits are important but often overlooked components of youth well-being especially in challenging times. Depression, anxiety, and insomnia were almost always present and dietary changes were significantly associated with the presence of them.

Keywords: Psychiatric morbidity, Depression, Anxiety, Insomnia, Diet, COVID-19, Youth, Mental Health, Coping, Eating habits

\section{Background}

Coronavirus disease (COVID-19) has become a potent destroyer of human health and the global economy in the twenty-first century and may be considered the most extraordinary global public health emergency nowadays. The World Health Organization (WHO) announced spreading of coronavirus rapidly across the globe, starting from China and becoming a pandemic by 11 March 2020. It has spread to 222 countries, affected over than 85 million people worldwide and killed over one million and half [1]. All continents announced confirmed cases of COVID-19. In Egypt, the first confirmed case was announced on 14 February 2020 [2].

Coronavirus disease (COVID-19) affects all segments of society worldwide. Despite that young people are less affected clinically by COVID-19 than adults, they are indirectly impacted by the pandemic [3]. Such a pandemic influenced the future of the young generation, the world's most valuable asset.

Although recently published evidence heavily outlined the physical complaints in the time of COVID-19, a paucity of research targeted psychological ones [4]. In this context, daily stressful events, prolonged home confinement, financial worries, violence, and over usage of the internet are reasons that could affect the mental health of youth during this critical period [5]. In addition, most activities that usually occupy youth's lives, such as schooling, social, and extracurricular activities with mates, have been disrupted. Putting all together, these will have broad-ranging influences on youth development and their mental health depending on their vulnerability and coping abilities in times of crisis [6]. These interruptions are expected to worsen or trigger mental illness, including anxiety, depression, and/or stressrelated symptoms.

Psychological state of adults in Egypt has been investigated in the time of COVID-19 [7]. However, to date, no detailed research has been carried out on psychiatric morbidity and eating habits in young people facing the pandemic. Hence, the current study aims to describe the real-time state of Egyptian youth's psychiatric morbidity, dietary habits, and coping methods during this pandemic and explore probable factors influencing them. It is an attempt to help in preserving the psychological wellbeing of the community.

\section{Methods \\ Study design and study population}

A descriptive cross-sectional study was performed using an online survey. Egyptian adolescents and young adults of both genders aged between 14 and 24 were reached through online advertisements on social media channels. Participants with a history of mental illness and debilitating physical disease were excluded. The survey was administered from 2 July to 23 July 2020.

Based on a prevalence rate of $58.0 \%$ for depression symptoms among Egyptian youth with a mean age of $20.18 \pm 1.50$ years [8], using $5 \%$ accepted degree of precision, $\alpha$ of 0.05 and power of $95 \%$, the minimum required sample size was 375 participants. The sample size increased up to 447 participants. The sample size was calculated using EPI-Info 2002 software [9].

\section{Measurement}

A data collection sheet was constructed to collect sociodemographic data, including age, gender, residence, and medical history of chronic physical illness. Additionally, some questions related to COVID-19 infection, such as financial loss due to the COVID-19 pandemic and whether any of the family members got infected by COVID-19, were included.

Based on a systematic review [10], questions related to dietary changes during the COVID-19 pandemic were added, such as bodyweight changes, the number of meals/day, changes of dietary habits, emotional eating, and night eating in addition to consumption of the beverage, caffeinated and energy drinks. Emotional eating was defined as the propensity to over-eat as a direct response to negative emotions such as anxiety, fear, or stress. Night eating habit was described as intake of at least $25 \%$ of one's total daily calories at night.

Coping methods among the participants were investigated by inquiry about the strategies used during the COVID-19 pandemic. These questions were conceptualized based on review of the literature [11-13]. Coping strategies were assessed by a question: "What do you do 
when the current pandemic burdens you?" 12 variables were used, each with 2 responses (no, yes). Coping methods were divided into 2 groups; positive (e.g., increase self-awareness regarding COVID-19, communication with other and seeking support, engagement in a hobby, exercise, and pray) and negative group (e.g., denial, tendency to stay alone, being aggressive and antisocial, smoking, consumption of hypnotics, consumption of caffeine, and substance abuse).

Depression was evaluated using the Arabic version of the Patient Health Questionnaire (PHQ-9) [14]. The PHQ-9 involves nine items representing the criterion symptoms for major depressive disorder by The Diagnostic and Statistical Manual of Mental Disorders, Fifth Edition (DSM-5) [15]. Participants were asked how many times they had been bothered by each symptom over the past 2 weeks, with response choices of not at all, several days, more than half the days, and nearly every day, scored as $0,1,2$, and 3 , respectively. Participants were categorized into four groups according to their scores, mild (5-9), moderate (10-14), moderately severe (15-19), and severe (20-27) [14].

For assessing anxiety symptoms, the Arabic version of the Generalized Anxiety Disorder Scale (GAD-7) was used [16]. The GAD-7 comprises 7 items with the same response options as PHQ-9. Although GAD-7 was originally developed as an indicator of generalized anxiety disorder, the GAD-7 operating characteristics are mostly as good for the other common anxiety disorders (e.g., panic disorder, social anxiety disorder, and posttraumatic stress disorder). GAD-7 scores were graded as 5-9 for mild, 10-14 for moderate, and 15-21 for severe [16] .

The validity and reliability of the Arabic versions of PHQ-9 and GAD-7 have been demonstrated among Saudi university students. Cronbach's alpha value for the internal consistency reliability was 0.857 and 0.763 for the Arabic versions of PHQ-9 and GAD-7, respectively [17].

Insomnia was evaluated using The Arabic version of the Insomnia Severity Index (ISI) [18]. ISI assesses insomnia depending on criteria by the International Classification of Sleep Disorders [19]. The ISI has seven items to evaluate the perceived severity of sleep initiating difficulties, staying asleep, and early morning awakenings, satisfaction with current sleeping patterns, intervention on daily functioning, and noticeability of impairment attributed to the sleep problem, as well as the degree of distress or anxiety caused by sleep problems. The scores for symptoms severity were $8-14$ for mild, 15-21 for moderate, and 22-28 for severe insomnia associated with significant daytime dysfunction [18]. The translated ISI showed adequate reliability and validity among the Arab population. Cronbach's alpha coefficient was 0.84 [20].

\section{Statistical analysis}

In order to explain the features of the study participants, frequency distribution and descriptive statistics (mean \pm standard deviation) were used. Bivariate analysis was conducted using the chi-square test to evaluate the participants in the sample for the occurrence or absence of symptoms of depression, anxiety and insomnia. The exact Fisher test was used when chi-square test was not applicable. Depression, anxiety, and insomnia symptoms were represented in the bivariate analysis as dichotomous variables (no, yes). Statistical testing was conducted using statistical package for social science (SPSS version 21.0) [21]. Two-tailed $p$ value $<0.05$ was considered statistically significant.

\section{Ethics approval and consent to participate}

Approval from the Ethics' Committee of High Institute of Public Health, Alexandria University, was obtained before conducting the current study. After explaining the study's purpose, all participants were provided with informed written online consent for participation and publication. For those who were less than 16 years old, informed written parental consent was obtained. The online survey was conducted anonymously to ensure the confidentiality of data. This work has been carried out in accordance with The Code of Ethics of the World Medical Association (Declaration of Helsinki) on Human participants.

\section{Results}

Participants were between 14 and 24 years old $(n=447)$ with mean age of $20.72 \pm 1.92$ years, $21.3 \%$ were adolescents between 14 and 19 years old $(n=95)$. Out of them, $70.2 \%$ were females and three-quarters (74.9\%) were urban residents. On the one hand, 9.4\% reported having a family member infected with COVID-19. On the other hand, approximately two-thirds of their families suffered from financial loss due to COVID-19 (61.7\%) (Table 1).

A minority of the participants (4.5\%) reported history of chronic physical illness. The participants experienced different grades of depression (80.5\%), anxiety (74\%), and insomnia $(73.8 \%)$. The most common positive coping methods practiced during COVID-19 pandemic were raising self-awareness regarding COVID-19, praying and communicating with others. Meanwhile, tendency to stay alone was the most common negative coping method (Table 1).

Regarding dietary changes during COVID-19, 37.4\% gained weight while $20 \%$ reported weight loss. Around half of the sample (49.9\%) had changes in their number 
Table 1 Characteristics of the study participants $(n=447)$

\begin{tabular}{|c|c|}
\hline Characteristics & $n(\%)$ \\
\hline \multicolumn{2}{|l|}{ Gender } \\
\hline Male & $\begin{array}{l}133 \\
(29.8)\end{array}$ \\
\hline Female & $\begin{array}{l}314 \\
(70.2)\end{array}$ \\
\hline
\end{tabular}

Age, years (mean $\pm S D=20.72 \pm 1.92)$

14-19

$95(21.3)$

20-24

352

(78.7)

Residence

Urban

335

(74.9)

Rural

112

(25.1)

Medical history of chronic physical illness

No

427

(95.5)

Yes

$20(4.5)$

Did any member of your family get infected with COVID-19?

No

Yes

Financial loss due to COVID-19

No

Yes

\section{5}

(90.6)

$42(9.4)$

171

(38.3)

276

(61.7)

Depression $($ mean $\pm S D=11.21 \pm 6.58)$

None-minimal

Mild

Moderate

Moderately severe

Severe

Anxiety (mean $\pm S D=9.31 \pm 6.30$ )

None-minimal

Mild

Moderate

Severe

Insomnia (mean $\pm S D=12.367 \pm 6.41$ )

No clinically significant insomnia

Mild to moderate insomnia

Moderate insomnia
87 (19.5)

106

(23.7)

115

(25.7)

$76(17.0)$

$63(14.1)$

116

(26.0)

127

(28.4)

104

(23.3)

100

(22.4)

117

(26.2)

161

(36.0)

133
Table 1 Characteristics of the study participants $(n=447)$

(Continued)

\begin{tabular}{ll}
\hline Characteristics & $\boldsymbol{n}(\%)$ \\
\hline & $(29.8)$ \\
Severe insomnia associated with significant impairments of & $36(8.1)$ \\
daytime functioning & \\
Coping methods ** & \\
Positive methods & \\
Raise self-awareness regarding COVID-19 & 403 \\
& $(90.2)$ \\
Praying & 374 \\
Communication with other and seeking support & $(83.7)$ \\
& 329 \\
Engagement in a hobby & $(73.6)$ \\
Exercise & 225 \\
& $(50.3)$ \\
Negative methods & 179 \\
Tendency to stay alone & $(40.0)$ \\
Being aggressive and antisocial & \\
Denial & \\
Smoking & 322 \\
Consumption of hypnotics & $(72.0)$ \\
\hline & $52(11.6)$ \\
Substance abuse & $69(15.4)$ \\
& $36(8.1)$ \\
& $22(4.9)$ \\
& $6(1.3)$ \\
\hline
\end{tabular}

COVID-19 coronavirus disease 2019

${ }^{* *}$ Multiple responses

of daily meals. Meanwhile, quarter of the sample (25.1\%) reported increased consumption of healthy food, while $24.2 \%$ had increase in consumption of junk food. Emotional and night eating emerged as new habits during the pandemic among $17.9 \%$ and $29.3 \%$ of the participants, respectively. A change in consumption of carbonated beverages was reported in $49 \%$ while $50.3 \%$ had changes in their consumption of caffeinated and energy drinks Table 2.

Depression symptoms were significantly associated with female gender $(P<0.001)$, adolescent age-group $(P$ $=0.002)$, having a family member infected with COVID$19(P=0.034)$, anxiety, and insomnia symptoms $(P<$ $0.001)$. Similarly, female gender $(P<0.001)$, adolescence $(P=0.002)$, a history of physical illness $(P=0.029)$, having a family member infected with COVID-19 $(P=$ $0.029)$, depression and insomnia symptoms $(P<0.001)$ were significantly associated with anxiety. Meanwhile, insomnia symptoms were significantly higher among females $(P=0.016)$, those with a history of physical illness $(P=0.028)$, had a family member infected with COVID$19(P=0.027)$, and presence of depression and anxiety $(P<0.001)$ (Table 3). 
Table 2 Dietary changes among the study participants during COVID-19 pandemic $(n=447)$

\begin{tabular}{|c|c|}
\hline Dietary changes & $n(\%$ \\
\hline \multicolumn{2}{|l|}{ Body weight change } \\
\hline No change & $190(42.5)$ \\
\hline Weight loss & $90(20.1)$ \\
\hline Weight gain & $167(37.4)$ \\
\hline \multicolumn{2}{|l|}{ Number of meals/day } \\
\hline No change & $224(50.1)$ \\
\hline Decreased & $120(26.8)$ \\
\hline Increased & $103(23.0)$ \\
\hline \multicolumn{2}{|l|}{ Changes of dietary habits } \\
\hline No change & $152(34.0)$ \\
\hline Poor appetite & $75(16.8)$ \\
\hline Increased consumption of junk food & $108(24.2)$ \\
\hline Increased consumption of healthy food & $112(25.1)$ \\
\hline \multicolumn{2}{|l|}{ Emotional eating } \\
\hline Not a habit at all & $251(56.2)$ \\
\hline A habit before COVID-19 & $116(26.0)$ \\
\hline A new habit during COVID-19 & $80(17.9)$ \\
\hline \multicolumn{2}{|l|}{ Night eating } \\
\hline Not a habit at all & $157(35.1)$ \\
\hline A habit before COVID-19 & 159 (35.6) \\
\hline A new habit during COVID-19 & $131(29.3)$ \\
\hline \multicolumn{2}{|l|}{ Consumption of carbonated beverage } \\
\hline No change & $228(51.0)$ \\
\hline Decreased & $131(29.3)$ \\
\hline Increased & $88(19.7)$ \\
\hline \multicolumn{2}{|c|}{ Consumption of caffeinated and energy drinks } \\
\hline No change & $222(49.7)$ \\
\hline Decreased & $96(21.5)$ \\
\hline Increased & $129(28.9)$ \\
\hline
\end{tabular}

Bodyweight changes are significantly associated with each of depression, anxiety, and insomnia $(P<0.001)$. Dietary changes in form of number of daily meals, changes of dietary habits, practicing emotional and night eating recently, increased consumption of carbonated beverage, and increased consumption of caffeinated and energy drinks were significantly associated with existence of depression, anxiety, and insomnia symptoms (Table 4).

\section{Discussion}

The COVID-19 pandemic may negatively influence mental health as a consequence of the distinctive blend of the public health crisis, social isolation and economic crises [22]. Measures to contain the virus have reshaped many facets of daily life, such as income, employment, schooling, and social interactions. Considering the direct impact of the virus and related consequences, researchers anticipated an increase in anxiety, depression, and their aftermath, including suicide [23]. Results of the current study revealed that depression and anxiety were almost always present with different severities. This high percentage agrees with a Chinese survey among adolescents aged 12-18; as symptoms of depression and anxiety during the COVID-19 outbreak were highly prevalent [24]. Similarly, in a study in Spain [25], during the first weeks of COVID-19 confinement, large numbers of students experienced moderate to extremely severe scores of anxiety and depression.

In comparing the results of the present study to other studies during COVID-19 pandemic, the current sample PHQ-9 mean score for depression (11.21 \pm 6.58) was higher than that of Lin's et al. [26], who studied 5641 Chinese individuals across the different age groups, as the average PHQ-9 score of their participants was $6.10 \pm$ 5.97. Also, in the same study, the anxiety scores assessed using GAD-7 had an average score of $4.97 \pm 5.25$ [26], while the current sample GAD-7 mean score was $(9.31$ \pm 6.30 ) which is higher.

Furthermore, with a mean ISI score of $12.36 \pm 6.41$, the prevalence of clinical insomnia was $73.8 \%$. This is much higher than reported before in a Chinese study; the average ISI score was $5.93 \pm 5.88$ and the prevalence of clinical insomnia was $20.05 \%$ [26]. While in other studies, insomnia was identified in $23 \%$ of 11,835 young adults [27]. Youth experienced distress, lack of energy, irregular naps, more screen time, and unrestricted online social media. A well-recognized feature of Egyptian sleep practice is biphasic sleep distributed in afternoon and late night bouts. Also, most of Egyptians eat the main meal of the day around mid-afternoon, and then nap in the late afternoon. In the evening, activity picks up again. The night bout of sleep begins sometime after midnight and ends after dawn [28]. These disruptive effects of social disorganization can affect bedtime, sleep quality, and sleep/wake patterns constancy directly or collectively [29].

Evidence found that depression and mental states among Arab adolescents are constructed and expressed differently than among adolescents in other cultures [30]. Culture may play a role in differences in frequency of disorders in different groups [31]. Also, socio-cultural factors may also play a part in how distress is expressed [32]. Hence, the differences across different population studies may emerge.

The present study showed significant associations between symptoms of depression, anxiety, and insomnia. Previous studies shown that symptoms of anxiety and depression are risk factors for insomnia and are associated and cyclically related [27, 33]. The relationship 
Table 3 Characteristics of the study participants by depression, anxiety, and insomnia symptoms $(n=447)$

\begin{tabular}{|c|c|c|c|c|c|c|}
\hline & \multicolumn{2}{|c|}{ Depression, $\boldsymbol{n}(\%)$} & \multicolumn{2}{|l|}{ Anxiety, $n(\%)$} & \multicolumn{2}{|l|}{ Insomnia, $n$ (\%) } \\
\hline & No 87 (19.5) & Yes $360(80.5)$ & No $116(26.0)$ & Yes 331 (74.0) & No 117 (26.2) & Yes 330 (73.8) \\
\hline \multicolumn{7}{|l|}{ Gender } \\
\hline Male & $50(37.6)$ & $83(62.4)$ & $59(44.4)$ & 74 (55.6) & 45 (33.8) & $88(66.2)$ \\
\hline Female & $37(11.8)$ & $277(88.2)$ & $57(18.2)$ & $257(81.8)$ & $72(22.9)$ & $242(77.1)$ \\
\hline$P$ value & \multicolumn{2}{|c|}{$<0.001 *$} & \multicolumn{2}{|c|}{$<0.001 *$} & \multicolumn{2}{|c|}{$<0.016^{*}$} \\
\hline \multicolumn{7}{|l|}{ Age, years } \\
\hline 14-19 & $8(8.4)$ & 87 (91.6) & $16(16.8)$ & 79 (83.2) & $20(21.1)$ & 75 (78.9) \\
\hline $20-24$ & $79(22.4)$ & $273(77.6)$ & $100(28.4)$ & $252(71.6)$ & $97(27.6)$ & $255(72.4)$ \\
\hline$P$ value & \multicolumn{2}{|c|}{$0.002 *$} & \multicolumn{2}{|c|}{$0.022 *$} & \multicolumn{2}{|c|}{0.201} \\
\hline \multicolumn{7}{|l|}{ Residence } \\
\hline Urban & 65 (19.4) & $270(80.6)$ & $85(25.4)$ & $250(74.6)$ & $86(25.7)$ & $249(74.3)$ \\
\hline Rural & $22(19.6)$ & $90(80.4)$ & $31(27.7)$ & $81(72.3)$ & $31(27.7)$ & $81(72.3)$ \\
\hline$P$ value & \multicolumn{2}{|c|}{0.956} & \multicolumn{2}{|c|}{0.630} & \multicolumn{2}{|c|}{0.676} \\
\hline \multicolumn{7}{|c|}{ Medical history of physical illness } \\
\hline No & $86(20.1)$ & $341(79.9)$ & $115(26.9)$ & $312(73.1)$ & $116(27.2)$ & $311(72.8)$ \\
\hline Yes & $1(5.0)$ & $19(95.0)$ & $1(5.0)$ & $19(95.0)$ & $1(5.0)$ & $19(95.0)$ \\
\hline$P$ value & \multicolumn{2}{|c|}{0.145} & \multicolumn{2}{|c|}{$0.029 *$} & \multicolumn{2}{|c|}{$<0.028 *$} \\
\hline \multicolumn{7}{|c|}{ Did your family get infected with COVID-19? } \\
\hline No & $84(20.7)$ & $321(79.3)$ & $111(27.4)$ & $294(72.6)$ & $112(27.7)$ & $293(72.3)$ \\
\hline Yes & $3(7.1)$ & $39(92.9)$ & $5(11.9)$ & $37(88.1)$ & $5(11.9)$ & $37(88.1)$ \\
\hline$P$ value & \multicolumn{2}{|c|}{$0.034 *$} & \multicolumn{2}{|c|}{$0.029 *$} & \multicolumn{2}{|c|}{$<0.027 *$} \\
\hline \multicolumn{7}{|c|}{ Financial loss due to COVID-19 } \\
\hline No & $31(18.1)$ & $140(81.9)$ & $50(29.2)$ & $121(70.8)$ & $49(28.7)$ & $122(71.3)$ \\
\hline Yes & $56(20.3)$ & $220(79.7)$ & $66(23.9)$ & $210(76.1)$ & $68(24.6)$ & $208(75.4)$ \\
\hline$P$ value & \multicolumn{2}{|c|}{0.575} & \multicolumn{2}{|c|}{0.212} & \multicolumn{2}{|c|}{$<0.348$} \\
\hline Insomnia & & & & & & \\
\hline No & $67(57.3)$ & $50(42.7)$ & $74(63.2)$ & $43(36.8)$ & - & - \\
\hline Yes & $20(6.1)$ & $310(93.9)$ & $42(12.7)$ & $288(87.3)$ & - & - \\
\hline$P$ value & & & & & & \\
\hline Anxiety & & & & & & \\
\hline No & 79 (68.1) & 37 (31.9) & - & - & 74 (63.8) & $42(36.2)$ \\
\hline Yes & $8(2.4)$ & 323 (97.6) & - & - & $43(13.0)$ & $288(87.0)$ \\
\hline$P$ value & & & & & & \\
\hline Depressior & & & & & & \\
\hline No & - & - & 79 (90.8) & $8(9.2)$ & $67(77.0)$ & $20(23.0)$ \\
\hline Yes & - & - & $37(10.3)$ & $323(89.7)$ & $50(13.9)$ & $310(86.1)$ \\
\hline$P$ value & & & & & & \\
\hline
\end{tabular}

COVID-19 coronavirus disease 2019

${ }^{*} P<0.05$

between sleep disturbance and depression is complex from the level of shared neurobiology to manifestation in clinical symptoms. Sleep duration and depressive symptoms appear to be related concurrently in a curvilinear manner [34]. Given the higher mean score of PHQ-9 in the current study, this might contribute to the higher prevalence of insomnia in comparison to other studies. Studies found that almost $75 \%$ of children and adolescents with depression manifested sleep disturbances, with over 50\% experiencing insomnia [35]. Social distance and isolation that follows longterm lockdowns can be a risk factor for anxiety and 
Table 4 Association between dietary changes during COVID-19 and depression, anxiety, and insomnia symptoms among the participants $(n=447)$

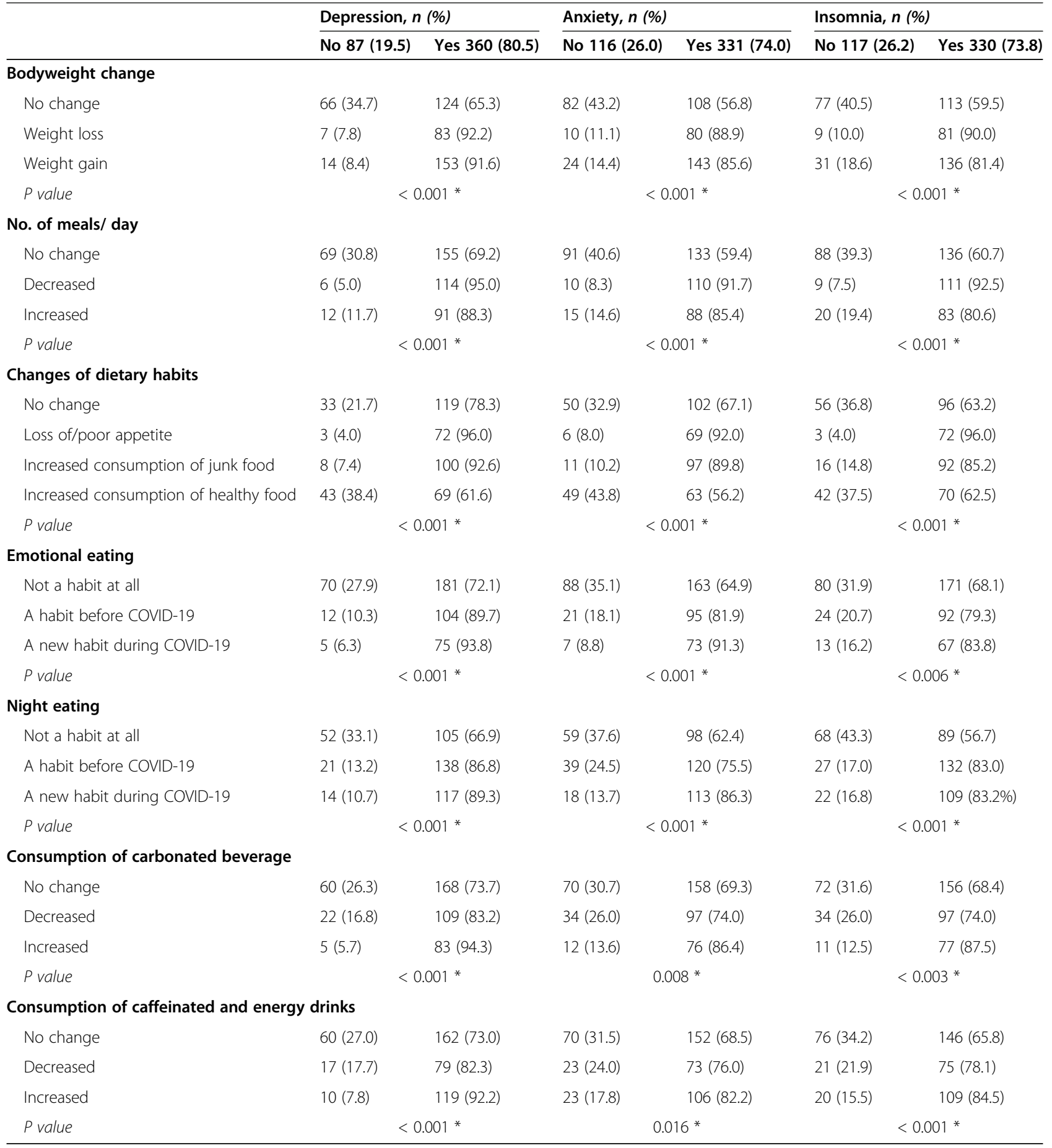

COVID-19 coronavirus disease 2019

${ }^{*} P<0.05$

mood disorders [36]. Variations in sleep during the COVID-19 pandemic may exacerbate or even contribute to psychopathology. Poor sleep can have a detrimental effect, leading to heightened vulnerability to mood and anxiety disorders [37].
An accumulating body of evidence indicated that females are at higher risk of depression and anxiety symptoms [24, 38], sleep problems [39], and negative psychological impact in general [7]. These results also matched the findings of this study, where female gender 
has significant associations with depression, anxiety, and insomnia. On the other hand, a study by Xie et al. reported no effect of gender [40].

Notably, youth between 14 and 19 had more severe scores on depression, anxiety but not insomnia, when compared to the older youth. Other studies revealed that younger persons reported higher psychological impacts $[7,26]$. These results are contradicting with a study Zhou et al. that found prevalence of symptoms of insomnia was higher among senior high school and college students [27]. The inverse correlation between respondents' age and the prevalence of depressed mood, anxiety, and insomnia was recorded in another study [41]. In the studied population, age has no significant association with insomnia. The timing and amount of sleep are largely determined by direct and interactive effects of gender, age, and sleeping arrangements [28].

It appears that the residence may give an idea about the circumstances in which the person lives and hence on his social conduct [42]. The result of the current study reveals that residence had no effect on association with psychopathology. This was in contrast to studies in China that found that depression and anxiety symptoms in cities were lower than in rural areas [24, 43]. A Greek study also found that people in urban areas were more vulnerable to sleep problems [39], while an Egyptian study found rural residency had a negative impact on mental health during COVID-19 [7]. This might be implied by the fact of urbanization of the countryside and ruralization of cities is taking place [44]. Considerably, the association of psychopathology and residence may change based on the population studied.

The mind and mental health are influenced by the health of the body. This is observed in the present study where young people with chronic physical illness had significantly higher anxiety and insomnia scores but not depression. El-Zoghby et al. reported that those with chronic illnesses are more susceptible to psychological distress [7]. Furthermore, some of them may perceive themselves with poor health and more liable to get diseased $[45,46]$. Even after controlling for confounders, strong cross-effects between physical and mental health are still valid [47].

Moreover, those who had a family member infected with COVID-19 had more significant distress in the form of depression, anxiety, and insomnia. These findings are corresponding to Cao et al., who found that having relatives or acquaintances with COVID-19 infection were risk factors for anxiety among Chinese undergraduate students [43]. Besides, all family members may have their COVID-19-related fears. Taken together, this response can lead to significant psychological distress for all family members, especially youth.
Economic recessions as during pandemic are accompanying with increased mental health problems for youth [22]. Family income has a significant impact on young people response to life stressors such as the COVID-19 crisis [48]. In a study among 7143 college students, family income stability was a protective factor against anxiety in the era of COVID-19 [43]. However, the results of the current study revealed that financial loss affected neither mood nor sleep. Although, low socioeconomic status is generally associated with high psychiatric morbidity, the nature of this association is not clear-cut [49]. Part of the difficulty is in disentangling other factors and their interactions, which are beyond the scope of current study.

Concerning changes in bodyweight during COVID-19 lockdown, $37.4 \%$ of the sample reported weight gain, $20.1 \%$ reported weight loss, while around half of the sample reported change in their number of daily meals. These are comparable to a study in Poland, in which around $30 \%$ and over $18 \%$ experienced weight gain and loss respectively, while over $43.0 \%$ and nearly $52 \%$ reported eating and snacking more [50]. Consistently, a study [51] on 3533 Italians found the sense of satiety and hunger altered for more than $50 \%$ of their population; $17.8 \%$ of them had less appetite, comparable to $16.8 \%$ of the sample. Phillipou et al. provided further support and reported increased restricting and binge eating behaviors among Australian population [52]. Notably, isolation leads to an increased sedentary behaviors and more physical inactivity which are expected to affect weight [53]. The excessive food exposure caused by the only freedom permitted was grocery shopping, which prompted people who were least successful in managing their diet to amplify the association between food consumption and feelings [41].

Furthermore, the current study is in line with an extensive body of evidence indicated that food consumption, eating patterns, food type, number of main meals, emotional and night, in addition to beverages consumption (caffeinated and carbonated), all were affected during confinement [54]. In addition to bodyweight, all described dietary changes have significant associations with depression, anxiety and insomnia. Previous studies reported increased scores of emotional and night eating in response to external stimuli with PHQ-9 and GAD-7 scores [55].

Pandemic-related anxiety may increase the difficulty of patients in managing their eating habits [56]. From another point of view, the consumption of palatable foods may have positive and reinforcing implications. The stress response can be sensibly controlled through its soothing effect [57]. However, the current study pointed out that around one-quarter of the sample improved their diet in consuming a more healthy diet and nearly 
the third decreased their intake of carbonated beverages. These findings were also reported by Di Renzo et al. but only $16.7 \%$ of their population improved their behaviors in the form of a decrease in consumption of snacks, savory food, carbonated, and sugary drinks [51].

Participants of both sexes reported various positive and negative coping strategies. In stress situation, coping mechanisms are used to control, mitigate, or tolerate stress and stressors such as those during COVID-19. A study involved 16 countries found that the most utilized coping strategies during COVID-19 were watching television for entertainment, social networking, listening to music, sleeping, performing household chores like cleaning and washing, eating well, and clearing/finishing piled-up work [58]. While the strategies used the most by participants of the current study were raising selfawareness regarding COVID-19, praying, communication with others, staying alone, and engagement in a hobby.

This study sheds important light on youth's psychiatric morbidity and dietary habits during the COVID-19 pandemic. Up to our knowledge, this study is one of the first studies to address such matters in Egypt and the Middle East. However, there is no study without limitations. The cross-sectional design of the current study limits the possibility of establishing temporality. Additionally, the psychological status was assessed using a selfadministered online survey. A systematic psychiatric evaluation through interviews of the participants would give a better assessment. Dietary habits and coping methods were assessed using designed questionnaires. Although these questionnaires were developed after a careful review of literature, the tools were new and could add to the limitations.

\section{Conclusion}

Psychiatric morbidity and dietary changes are evident in young people during COVID-19 pandemic. Psychological well-being and dietary habits are important but often overlooked components of youth well-being especially in challenging times. Results of the current study highlighted the presence of depression, anxiety, insomnia during the COVID-19 pandemic. This study also gave an idea about dietary changes during the pandemic and various coping methods. These findings suggest that, as the pandemic continues to evolve, it is important to continue the monitoring of young people well-being. More controlled studies are needed to assess the causality between stress during the pandemic, mental health impacts and dietary changes. In future studies, assessment of dietary habits and coping methods using validated questionnaires would be of an added value. Putting it all together, timely action could mitigate the current negative state and improve mental health and well-being.
Developing a strategy targeting fulfilling these unmet needs is just one way to help our youth deal with the uncertain coming days.

\section{Abbreviations}

COVID-19: Coronavirus disease 2019; DSM: Diagnostic and Statistical Manual of Mental Disorders; GAD-7: Generalized anxiety disorder scale; ISI: Insomnia Severity Index; PHQ-9: Patient Health Questionnaire; WHO: The World Health Organization

\section{Acknowledgements}

We would like to show our gratitude to Dr. Mamdouh Wahba, the President of the Egyptian Society for Adolescent Medicine (ESAM) and the current Vice President of the International Association for Adolescent Health (IAAH). This study would never have been possible without his support. We are indebted to all the participants for their cooperation.

\section{Authors' contributions}

RA, NF, and AK all contributed in conceiving the presented idea, developed the methodology and data collection; drafted the article; and helped shape the research, analysis, and manuscript. RA is the corresponding author, designed the online survey, extracted the data, was responsible for the literature search, and has a major contribution in writing the manuscript, critical revision, and verifying of the final version of the article after meeting the journal's standards. NF obtained the ethical approval, performed computations, verified the analytical methods, data tabulation, contributed to manuscript writing, editing, and critical revision. AK data presentation and interpretation, contributed to manuscript writing, editing, and critical revision. All authors have read and approved the manuscript.

Funding

No funding was received for this particular research.

\section{Availability of data and materials}

Data is available upon request.

\section{Ethics approval and consent to participate}

Ethical approval was obtained before conducting the study from the Ethics' Committee of High Institute of Public Health, Alexandria University,

Alexandria, Egypt (ethics committee reference number: not applicable). After explaining the study's purpose, all participants were provided with informed written online consent for participation and publication. For those who were less than 16 years old, informed written parental consent was obtained.

\section{Consent for publication}

All participants and parents of those who were less than 16 years old provided an informed written consent for publication.

\section{Competing interests}

The authors declare that they have no competing interests.

\section{Author details}

${ }^{1}$ Mamoura Psychiatric Hospital, General Secretariat of Mental Health and Addiction Treatment, El Nabawy El Mohandes Street, Mamoura, Alexandria 21912, Egypt. ${ }^{2}$ Family Health Department, High Institute of Public Health, Alexandria University, Alexandria, Egypt. ${ }^{3}$ Community Health Nursing Department, Faculty of Nursing, Alexandria University, Alexandria, Egypt.

Received: 1 December 2020 Accepted: 15 January 2021

Published online: 01 February 2021

\section{References}

1. World Health Organization (WHO). Coronavirus Disease (COVID-19) Pandemic Information Center: Numbers at a glance. Available at: https:// www.who.int/emergencies/diseases/novel-coronavirus-2019. Accessed 7 Jan 20212020.

2. Egypt Today Magazine. Egypt announces first Coronavirus infection. Available at: https://www.egypttoday.com/Article/1/81641/Egyptannounces-first-Coronavirus-infection. Accessed 17 Aug 2020.

3. Clark H, Coll-Seck AM, Banerjee A et al (2020) After COVID-19, a future for the world's children? Lancet 396:298-300 
4. Torales J, O'Higgins M, Castaldelli-Maia JM et al (2020) The outbreak of COVID-19 coronavirus and its impact on global mental health. Int J Soc Psychiatr:0020764020915212

5. Guessoum SB, Lachal J, Radjack R et al (2020) Adolescent psychiatric disorders during the COVID-19 pandemic and lockdown. Psychiatry Res 291:113264

6. Golberstein E, Wen H, Miller BF (2020) Coronavirus disease 2019 (COVID-19) and mental health for children and adolescents. JAMA Pediat 174(9):819-820

7. El-Zoghby SM, Soltan EM, Salama HM (2020) Impact of the COVID-19 pandemic on mental health and social support among adult Egyptians. J Commun Health:1

8. Effat S, Azab H, Aly H et al (2019) The relationship between anxiety, depression, and problematic internet use among a sample of university students in Egypt. Sohag Med J 23:154-165

9. CDC (2002) EPI Info -Database and statistics software for public health professionals. Centre for Disease Control and Prevention, Atlanta

10. O'Neil A, Quirk SE, Housden S et al (2014) Relationship between diet and mental health in children and adolescents: a systematic review. Am J Public Health 104:e31-e42

11. Garcia C (2010) Conceptualization and measurement of coping during adolescence: a review of the literature. J Nurs Scholar 42:166-185

12. Smith C, Carlson BE (1997) Stress, coping, and resilience in children and youth. Soc Serv Rev 71:231-256

13. Hamdan-Mansour AM, Al Badawi T, Haourani E et al (2013) Depression, psychological distress and coping skills among patients diagnosed with type-II Diabetes Mellitus. Life Sci J:10

14. Spitzer R, Kroenke K, Williams J (1999) Validation and utility of a self-report version of PRIME-MD: the PHQ primary care study. Primary Care Evaluation of Mental Disorders. Patient Health Questionnaire. JAMA 282:1737-1744

15. American Psychiatric Association. Diagnostic and statistical manual of mental disorders. 5th 'edition'. Washington, DC, 2013.

16. Spitzer R, Kroenke K, Williams J et al (2006) A brief measure for assessing generalized anxiety disorder: the GAD-7. Arch Intern Med 166:1092-1097

17. AlHadi AN, AlAteeq DA, Al-Sharif E et al (2017) An arabic translation, reliability, and validation of Patient Health Questionnaire in a Saudi sample. Ann Gen Psychiatry:16

18. Bastien C, Vallières A, Morin C (2001) Validation of the Insomnia Severity Index as an outcome measure for insomnia research. Sleep Med 2:297-307

19. American Sleep Disorders Association (1997) International classification of sleep disorders: diagnostic and coding manual reR. Ameri-can Sleep Disorders Association, MA

20. Suleiman $\mathrm{KH}, \mathrm{BC}$ Y (2011) Translating the insomnia severity index into Arabic. J Nurs Scholar 43:49-53

21. IBM Corp. IBM SPSS statistics version 21. IBM SPSS Statistics for Windows, 21 0 edition. Armonk, 2012.

22. Golberstein E, Gonzales G, Meara E (2019) How do economic downturns affect the mental health of children? Evidence from the National Health Interview Survey. Health Econ 28:955-970

23. Gavin B, Lyne J, McNicholas F (2020) Mental Health and the COVID19 Pandemic. Irish J Psychol Med:1-7

24. Zhou S-J, Zhang L-G, Wang L-L et al (2020) Prevalence and socio-demographic correlates of psychological health problems in Chinese adolescents during the outbreak of COVID-19. Eur Child Adolesc Psychiatr:1-10

25. Odriozola-González P, Planchuelo-Gómez Á, Irurtia MJ et al (2020) Psychological effects of the COVID-19 outbreak and lockdown among students and workers of a Spanish university. Psychiatr Res 290:113108

26. Lin L-Y, Wang J, Ou-Yang X-Y, et al. The immediate impact of the 2019 novel coronavirus (COVID-19) outbreak on subjective sleep status. Sleep Medicine 2020.

27. Zhou SJ, Wang LL, Yang R, Yang XJ, Zhang LG, Guo ZC, Chen JX (2020) Sleep problems among Chinese adolescents and young adults during the coronavirus-2019 pandemic. Sleep Med 74:39-47

28. Worthman CM, Brown RA (2007) Companionable sleep: social regulation of sleep and cosleeping in Egyptian families. J Fam Psychol 21:124

29. Becker SP, Gregory AM (2020) Editorial perspective: perils and promise for child and adolescent sleep and associated psychopathology during the COVID-19 pandemic. J Child Psychol Psychiatr Allied Discip 61:757-759

30. Obermeyer CM, Bott S, Sassine AJ (2015) Arab adolescents: health, gender, and social context. J Adolesc Health 57:252-262

31. Tanaka-Matsumi J. Abnormal Psychology and Culture. 2001

32. Anthony J, Sartorius N, Jablensky A et al (1985) Cross-cultural studies of depressive disorders: An overview. Cult Depress 16:299
33. Jansson M, Linton SJ (2006) The role of anxiety and depression in the development of insomnia: cross-sectional and prospective analyses. Psychol Health 21:383-397

34. Asaad T, Taha GR, Abu-Ella I et al (2014) Sleep profile in a sample of Egyptian school-aged children with depression. Middle East Curr Psychiatr 21:63-71

35. Yorbik O, Birmaher B, Axelson D, Williamson DE, Ryan ND (2004) Clinical characteristics of depressive symptoms in children and adolescents with major depressive disorder. J Clin Psychiat. 65(12):1654-1659

36. Sani G, Janiri D, Di Nicola M et al (2020) Mental health during and after the COVID-19 emergency in Italy. Psychiatr Clin Neurosci 74:372-372

37. Gregory AM, Sadeh A (2016) Annual research review: sleep problems in childhood psychiatric disorders-a review of the latest science. J Child Psychol Psychiatr 57:296-317

38. Oosterhoff B, Palmer CA, Wilson J et al (2020) Adolescents' motivations to engage in social distancing during the COVID-19 pandemic: associations with mental and social health. J Adolesc Health 67:179-185

39. Voitsidis P, Gliatas I, Bairachtari V et al (2020) Insomnia during the COVID-19 pandemic in a Greek population. Psychiatr Res:113076

40. Xie X, Xue Q, Zhou Y, Zhu K, Liu Q, Zhang J, Song R (2020) Mental health status among children in home confinement during the coronavirus disease 2019 outbreak in Hubei Province. China. JAMA Pediat 174(9):898-900

41. Di Renzo L, Gualtieri P, Cinelli G et al (2020) Psychological Aspects and Eating Habits during COVID-19 Home Confinement: Results of EHLC-COVID19 Italian Online Survey. Nutrients:12

42. Short SE, Mollborn S (2015) Social Determinants and Health Behaviors: Conceptual Frames and Empirical Advances. Curr Opin Psychol 5:78-84

43. Cao W, Fang Z, Hou G et al (2020) The psychological impact of the COVID19 epidemic on college students in China. Psychiatr Res 287:112934

44. Daigaku KG. Keio University symposia for life science and medicine: Springer, 1999.

45. Mazza C, Ricci E, Biondi S et al (2020) A nationwide survey of psychological distress among italian people during the COVID-19 pandemic: immediate psychological responses and associated factors. Int J Environ Res Public Health 17:3165

46. Hatch R, Young D, Barber V et al (2018) Anxiety, depression and post traumatic stress disorder after critical illness: a UK-wide prospective cohort study. Crit Care 22:310

47. Ohrnberger J, Fichera E, Sutton M (2017) The relationship between physical and mental health: A mediation analysis. Soc Sci Med 195:42-49

48. Almeida DM, Neupert SD, Banks SR et al (2005) Do daily stress processes account for socioeconomic health disparities? J Gerontol Series B 60:534-S39

49. Lorant $V$, Deliège $D$, Eaton $W$ et al (2003) Socioeconomic inequalities in depression: a meta-analysis. Am J Epidemiol 157:98-112

50. Sidor A, Rzymski P (2020) Dietary Choices and Habits during COVID-19 Lockdown: Experience from Poland. Nutrients 12:1657

51. Di Renzo L, Gualtieri P, Pivari F et al (2020) Eating habits and lifestyle changes during COVID-19 lockdown: an Italian survey. J Transl Med 18:229

52. Phillipou A, Meyer D, Neill E et al (2020) Eating and exercise behaviors in eating disorders and the general population during the COVID-19 pandemic in Australia: Initial results from the COLLATE project. Int J Eat Disord 53:1158-1165

53. Rundle AG, Park Y, Herbstman JB et al (2020) COVID-19-related school closings and risk of weight gain among children. Obesity 28:1008-1009

54. Ammar A, Brach M, Trabelsi K et al (2020) Effects of COVID-19 home confinement on eating behaviour and physical activity: results of the ECLBCOVID19 International Online Survey. Nutrients 12

55. Papandreou C, Arija V, Aretouli E et al (2020) Comparing eating behaviours, and symptoms of depression and anxiety between Spain and Greece during the COVID-19 outbreak: Cross-sectional analysis of two different confinement strategies. Eur Eat Disord Rev

56. Fernández-Aranda F, Casas M, Claes L et al (2020) COVID-19 and implications for eating disorders. Eur Eat Disord Rev 28:239

57. Parylak SL, Koob GF, Zorrilla EP (2011) The dark side of food addiction. Physiol Behav 104:149-156

58. Aga SS, Khan MA, Nissar SS et al (2020) Assessment of mental health and various coping strategies among general population living under imposed COVIDlockdown across world: a cross-sectional study. Ethics Med Public Health:100571

\section{Publisher's Note}

Springer Nature remains neutral with regard to jurisdictional claims in published maps and institutional affiliations. 